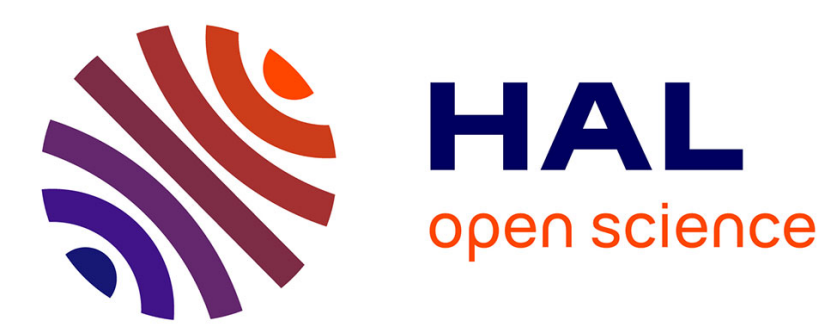

\title{
Ballistic simulation of Ratchet effect in antidot lattices patterned on graphene
}

Luca Pierantoni, Davide Mencarelli, Fabio Coccetti, Tullio Rozzi

\section{To cite this version:}

Luca Pierantoni, Davide Mencarelli, Fabio Coccetti, Tullio Rozzi. Ballistic simulation of Ratchet effect in antidot lattices patterned on graphene. European Microwave Week (EuMW), Sep 2015, Paris, France. hal-01238026

\section{HAL Id: hal-01238026 https://hal.science/hal-01238026}

Submitted on 4 Dec 2015

HAL is a multi-disciplinary open access archive for the deposit and dissemination of scientific research documents, whether they are published or not. The documents may come from teaching and research institutions in France or abroad, or from public or private research centers.
L'archive ouverte pluridisciplinaire HAL, est destinée au dépôt et à la diffusion de documents scientifiques de niveau recherche, publiés ou non, émanant des établissements d'enseignement et de recherche français ou étrangers, des laboratoires publics ou privés. 


\title{
Ballistic simulation of Ratchet effect in antidot lattices patterned on graphene
}

\author{
Luca Pierantoni $^{1,2}$, Davide Mencarelli ${ }^{1,2}$, Fabio Coccetti $^{3}$, Tullio Rozzi ${ }^{1}$ \\ ${ }^{1}$ Università Politecnica delle Marche Ancona, Italy, ${ }^{2}$ Istituto Nazionale di Fisica Nucleare (INFN-LNF) Frascati, Italy \\ ${ }^{3}$ CNRS, LAAS, F-31400 Toulouse, France; Univ de Toulouse, LAAS, F-31400 Toulouse, France, \\ 1.pierantoni@univpm.it
}

\begin{abstract}
In this contribution, we investigate the mechanism governing the Ratchet effect in patterned monolayer graphene, at the ballistic nanoscale. Still smaller than currently achievable and manifacturable devices, the simulated structures serve to exemplify the dependence of charge scattering on the arrangement of lattice defects, i.e. clusters of atomic vacancies of triangular shape. The ballistic Ratchet effect is seen as cumulative multimode scattering of carriers in correspondence of the lattice discontinuities. An atomistic model, based on the Scattering Matrix method and making use of TB approximation, has been applied. The latter, in contrast with continuum models, like Dirac or Kubo-Drude derived formulas, is capable of describing abrupt discontinuities at sub-micrometric scales, where graphene is likely to preserve its outstanding properties. We believe that this work is a first step in the direction of engineering and design of devices based on the ballistic Ratchet effect, like RF and THz detectors.
\end{abstract}

Keywords-Graphene, Ratchet effect, ballistic electronics, RF and $\mathrm{THz}$ detector, antidot lattice.

\section{INTRODUCTION}

One of the most emblematic phenomena based on ballistic transport is the Ratchet effect, originally introduced by R. Feynman in 1963 [1]. In general terms, the ratchet effect can be seen as a collective motion of particle systems in a preferential direction, under external excitation, in presence of spatially-asymmetric obstacles or perturbations. A basic discussion of the Ratchet effect focuses on possible violation of the second law of Thermodynamics, to which several explanations follows of why such idea fails. As expectable, an external force, or energy supply, is always needed, for the Ratchet effect to be sustained. A strong analogy with photovoltaic effect, based on crystal asymmetry at a microscopic level, can be noticed [2].

Important examples of "Ratchet devices" are given by i) transport of charge in two-dimensional electron gas (2DEG) at surface heterostructures, with nano-patterning of asymmetric defects or with space-dependent potential [3], and ii) biological molecular transport [4], where the Brownian random molecular motion can be exploited to achieve a linear displacement, or a directional rotation. In this case, the role played by thermal fluctuation in generating force and motion is a key enabling mechanism for chemo-mechanical transduction by motor proteins.

In the present work, we develop a theoretic analysis, propaedeutic to the optimization of the photogalvanic current induced by a high frequency radiation in monolayer graphene [5-7], patterned with asymmetric nano-defects. Specifically, the latter are given by clusters of atomic vacancies of triangular shape. In practice, these could be obtained by forming a periodic arrangement of triangular perforations in a graphene sheet. The above structure can be referred as a graphene antidot lattice, in analogy with standard antidot lattices in 2DEG [8,9].

We propose a completely ballistic picture of the Ratchet effect, where the main contribution to the collective and cumulative charge displacement is due to ballistic scattering. Numerical simulations are performed in the framework of the Scattering Matrix (SM) approach, described elsewhere [10], which is conceptually analogous to the non-linear Green's function formalism (NEGF). The main difference is that it provides a convenient description of the graphene lattice in terms of propagating and evanescent modes, much similar to the standard analysis of periodically loaded microwavewaveguides.

The SM method may allow simulation of large structures by a modular approach, owing to the possibility to easily connect the multimodal S-matrices describing smaller parts composing the entire structure.

Nevertheless, the above description is made numerically possible by the choice of a sub-micrometric size of the system under analysis. In fact, simulation of coherent transport in graphene nanostructures become rapidly prohibitive as the computational domain increases. To give an idea, a simple graphene square of $10 \times 10 \mathrm{~nm}^{2}$ consists of almost $4 \times 10^{3}$ carbon atoms, where the charge wavefunction must be sampled.

It is remarked that the present ballistic approach relies, for consistence with practical devices, on the long ballistic length, up to $1 \mu \mathrm{m}$, observable and achievable in monolayer graphene.

\section{SiMULATION OF CHARGE SCATTERING}

Triangular holes are analysed as paired defects in monolayer graphene, as depicted in Fig. 1(a). A concept device, acting as a RF detector or sensor, may involve periodic replication of 
these defects along the y-direction, in a multi-finger configuration, like it is shown in Fig. 1(b).

The shape of the holes affects the charge scattering and changes the charge distribution induced by an external applied voltage. The latter is given by a plane-wave impinging on the plane of graphene in the normal direction: the electric field $E$ is assumed as polarized along the y-axis, in order to promote charge displacement in the $\mathrm{x}$-direction, and subsequent collision with the holes.

Owing to their long mean free path, charges scattered in the $\mathrm{x}$ direction by the triangular defects are likely to reach the edges of graphene before the occurrence of other scattering events, like collisions with other carriers or impurities. Thus, charges are collected to the lateral metal contacts (see Fig. 1), and can give rise to a current flow. The spatial asymmetry of defects in the $\mathrm{x}$-direction implies a preferred direction for the current flow, namely in the positive $x$-direction due to the triangle orientation, regardless of the sign of $E_{y}$ : this fact can easily explain the rising of the electronic ratchet transport. The same principle can be used for creation of detectors operating at room temperature in the microwave/terahertz radiation range [11].

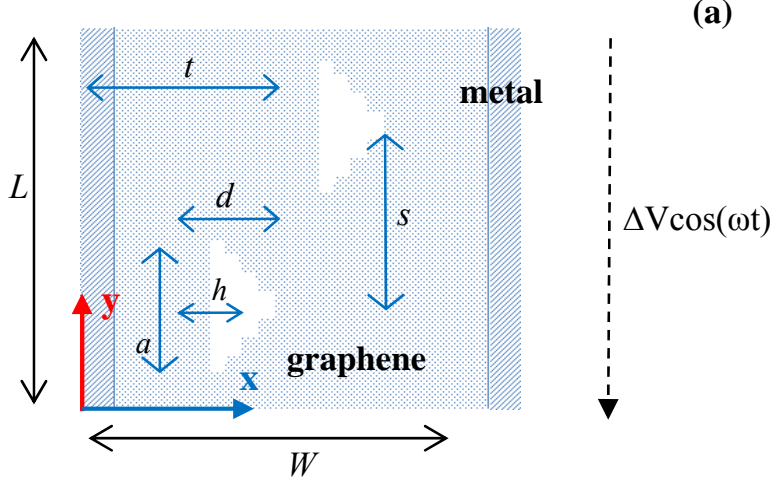

(b)

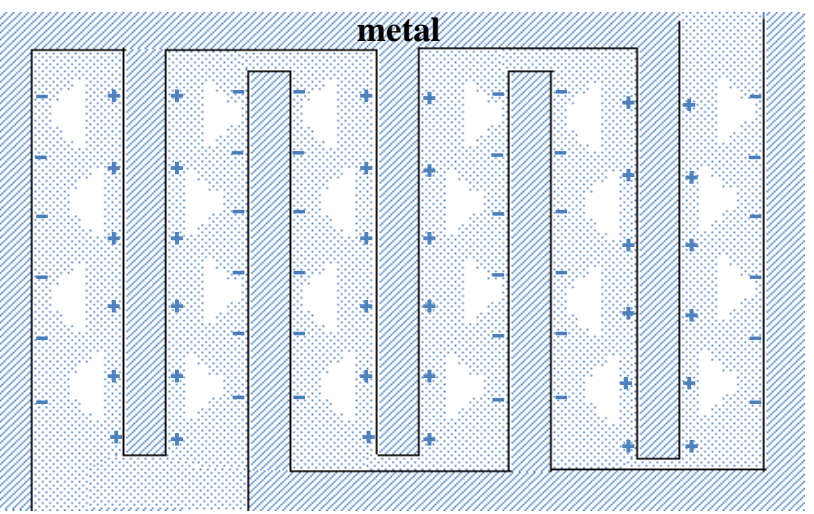

Fig. 1. a) Triangular vacancy defect in paired configuration. b) Interdigital metal structure on antidote graphene lattice with triangular holes.

Importantly, the SM approach avoids the use of a Dirac-like continuum description of charge transport: the presence of abrupt discontinuities, i.e. the edges of the holes, implies scattering between the two Dirac points of graphene, and makes a single "valley" picture potentially not reliable. The Dirac approach also assumes the approximation of linear bands of bulk graphene: in order to capture effects of the atomic structure, including the influence of defect boundaries, and to provide a realistic description of the band structure, we need to go beyond the simple Dirac fermion picture.

In a few words, the SM method [10] starts from a particular formulation of the Schrödinger Hamiltonian $H$ of graphene sheet of finite width (ribbon), arising from the tight-binding (TB) approach:

$$
H \psi=H_{l} \psi_{l}+H_{0} \psi_{0}+H_{r} \psi_{r}=E \psi
$$

where $H_{1}\left(H_{\mathrm{r}}\right)$ denotes the hopping energy matrix of the periodic unit-cell of the graphene ribbon to the previous one from the left (right), $E$ is the charge energy, $H_{0}$ contains the atomic self-energies and the self-generated/external potential, and $\psi_{1}, \psi_{0}, \psi_{r}$, are the unknown eigenstates of three consecutive unit-cells, expanded in a set of localized "atomic" samples. The above approach makes use of well-definite conditions that ensure numerical consistency: all electronic states are normalized to carry the same current (from Landauer condition [10]), and the excitation section ( $y=0$ and $y=L)$ are properly set as transparent, i.e. perfectly absorbing.

At microwave frequency, and in the low $\mathrm{THz}$ range, the time scale of the electromagnetic field is much larger than the time needed to the charges to reach the discontinuities and to be scattered from these to the lateral electrodes. Thus, quasi static approximation is largely acceptable.

In order to understand the ballistic mechanisms for ac detection, we consider a suitable situation where a small quasi-static voltage is applied across the graphene unit-cell of Fig. 1(a), along the y-axis. Our aim is to show that the charge injected by the external voltage tends to accumulate in one of the two edges $(x=0, x=W)$, depending on the spatial position of the holes. We neglect the charge interaction, and consider the charge distribution slightly deviated by its equilibrium state, as we are looking for the incremental charge induced by the small potential perturbation.

In the present framework, the lateral metal contacts are replaced by Dirichlet conditions for the electron wavefunctions. More rigorously, absorbing boundaries, mediated by the metal-graphene discontinuity, could be assumed, but this is largely beyond the purpose of the present work. Figure 2 shows an example of simulated distribution $|\psi|^{2}$ - namely charge per unit area and per unit energy, after Landauer normalization and multiplication by the unit charge $e$-which is obtained injecting charges from top of the region of Fig 1(a), at energy $\mu_{c}$.

The above implies summation over all the electronic state available at the Fermi level, and the sum is normalized to its peak value.

$$
|\psi|^{2}=\sum_{i=1: N}\left|\psi_{i}\right|^{2}
$$


where $i$ indicate electronic channels. Note, in Fig. 2(a,b), that strong charge concentrations are present along the perimeter of the hole discontinuities, and in particular in the vertices of the triangles.

The dc voltage across the unit cell, in the y-direction, mimics the $E_{y}$ polarized plane wave impinging on graphene.

This voltage can varied continuously in the interval from $-\Delta \mathrm{V}$ to $\Delta \mathrm{V}$, with $\Delta \mathrm{V}=\left|E_{y}\right| \cdot L$ and the resulting charge distribution can mediated in this interval, in order to reproduce an average charge distribution over a period $2 \pi / \omega$ of the electromagnetic excitation:

$$
Q=e \int_{\mu_{c}}^{\overline{\mu_{c}+v} \sum_{i=1: N}\left|\psi_{i}\right|^{2}}
$$

where $Q$ is charge for unit area, and the averaging is taken over $-\Delta \mathrm{V}<v<\Delta \mathrm{V}$. As it is shown in the above equation, only contribution of states near the Fermi level is retained. An example is shown in Fig. 3.

(a)

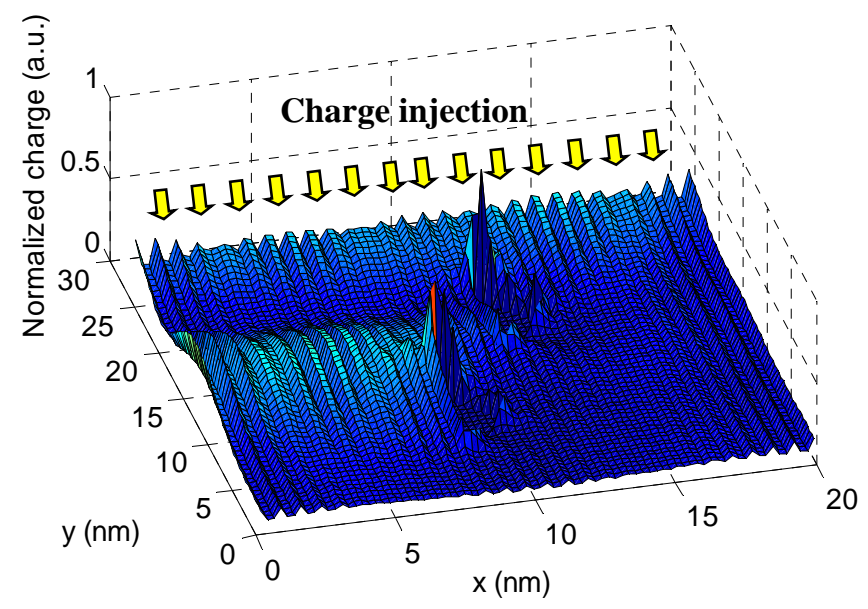

(b)
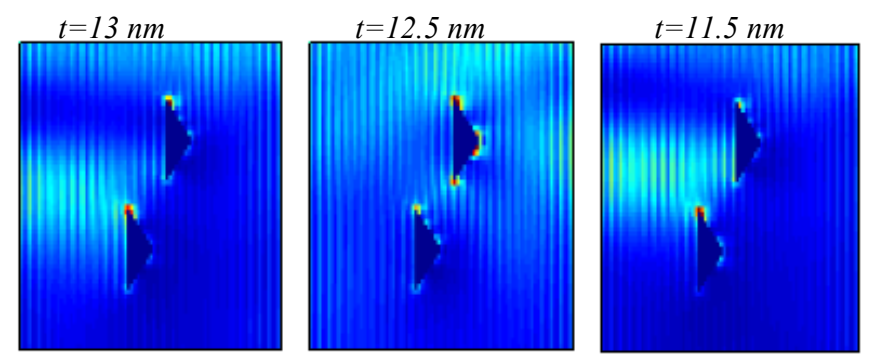

Fig. 2. a) Normalized charge distribution $|\psi|^{2}$ upon injection of electrons from top of the region of Fig 1(s). Here, $\mu_{c}=0.22 \mathrm{eV}$, with $d=3 \mathrm{~nm}, a=7 \mathrm{~nm}, h=2 \mathrm{~nm}, s=9 \mathrm{~nm}, t=13 \mathrm{~nm}$. b) Top view of surface charge density $Q$, with same parameters as a), but varying x: $t=13 \mathrm{~nm}$, $t=12.5 \mathrm{~nm}$, and $t=11.5 \mathrm{~nm}$.
Q (a.u.)

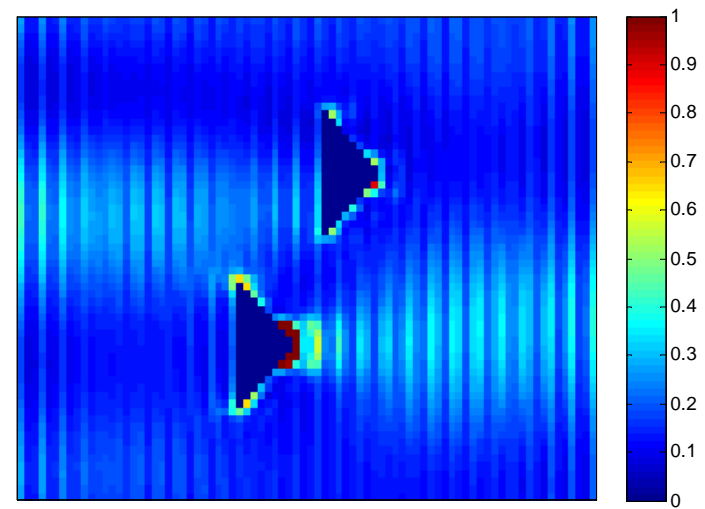

Fig. 3. Top view of surface charge density $Q$, with same parameters as Fig. 2, but varying $\mathrm{x}$ : a) $t=13 \mathrm{~nm}$, b) $t=12.5 \mathrm{~nm}$, c) $t=11.5 \mathrm{~nm}$.

\section{DISCUSSION}

At a small scale, namely few tens of nanometers, the Ratchet effect, seen as cumulative scattering and reflection of charges, is slightly visible, because the number of electronic channels is relatively small, for the typical charge density of undoped graphene. Here, a Fermi energy $\mu_{c}$ of $0.22 \mathrm{eV}$ with respect to the Dirac point, has been assumed, corresponding to a density of states $n_{s}=2 \mu_{c} / \pi \hbar^{2} \mathrm{VF}^{2}$.

Moreover, the size of the hole discontinuities is comparable with the wavelength of the majority of the propagating (electronic) modes. Thus, electrons see relatively small defects and the scattering is described by wave diffraction, instead of multiple reflection. This fact makes the charge distribution, after perturbation by the incident electromagnetic field, very sensitive to the geometry and position of the defects, preventing from the possibility to have a clear parametric trend. Incidentally, we note that the situation is quite similar to the case of three-terminal ballistic junctions, made of three-branch graphene nanoribbons (GNRs), that was recently analyzed [12], and that reproduces, in fact, a sort of triangular asymmetry. As a result, the $\mathrm{Y}$ ballistic junctions was shown to feature nonlinear and asymmetric transport properties. In practice, the central branch of a Y-shaped device, at zero voltage, was found to support current flow when the other two branches are subjected to equal voltages of opposite sign $[13,14]$.

As a further improvement of the present analysis, the Dirichlet boundary conditions at $\mathrm{x}=0$ and $\mathrm{x}=\mathrm{W}$ will be replaced by "impedance" boundaries, which means charge absorption with partial reflection due to the local band distortion at the metal transition. This kind of analysis provides, automatically, the net dc current between the lateral contacts, which are kept at zero dc voltage.

It is remarked that, even if charge asymmetry induced by external field constitutes a significant parameter, it must be weighted by the amount of reflection of the charge injected in 
the unit cell containing the holes. A high reflection coefficient means lower charge penetration, and decrease of the mean free path, which are likely to reduce the Ratchet effect.

Authors are currently working on i) the present TB approach, and on ii) the development of a full-wave time-domain solver, based on the transmission line matrix (TLM) method, aimed at modeling the combined electromagnetics-quantum transport in the ballistic regime, as in [15]. In the building of ii), a critical point arises from the presence of huge "aspect ratio" (maximum/minimal geometrical dimensions) in the $3 \mathrm{D}$ domain. To overcome this problem, a dedicated "subgridding" technique is under construction.

\section{CONCLUSION}

In this contribution, we presented a rigorous analysis and simulation of charge scattering by non-centrosymmetric defects patterned on graphene lattice. Precisely, these defects are due to paired holes of triangular shape. The analysis shows that charge distribution, subsequent to charge injection, strongly depends on the geometry of the defects, in particular their spatial position. Numerically, we are dealing with multimode (electronic) waveguides: in the ballistic scale, this multimodality is believed to be a direct explanation of the Ratchet effect, i.e. the raising of a directional dc current after excitation by harmonic waves. Current work is being done in order to enlarge the computational domain, and make simulations suitable for real devices employing the ballistic Ratchet effect, like RF detectors.

\section{ACKNOWLEDGEMENTS}

This work has been partially supported by the European Project, "Carbon Based Smart Systems for Wireless Applications”, (NANO-RF, n. 318352).

\section{REFERENCES}

[1] R.P. Feynman, R.B. Leighton, M. Sands, "The Feynman Lectures on Physics," Vol. 1, Chap. 46, Addison Wesley, Reading, MA, 1963.

[2] A.M. Glass, D. Linde, and T.J. Negran, "High-voltage bulk photovoltaic effect and the photorefractive process in $\mathrm{LiNbO}_{3}$ ", Appl. Phys. Lett. 25, 233, 1974,

[3] W. Weber, L.E. Golub, S.N. Danilov, J. Karch, C. Reitmaier, B. Wittmann, V.V. Bel'kov, E.L. Ivchenko, Z.D. Kvon, N.Q. Vinh, A. F.G. van der Meer, B. Murdin, and S.D. Ganichev, "Quantum ratchet effects induced by terahertz radiation in GaN-based twodimensional structures", Phys. Rev. B 77, 245304, 200

[4] R. D. Vale, and F. OOSAWA, "Protein motors and Maxwell's demon: does mechanochemical transduction involve a thermal Ratchet?", Adv. Biophys., Vol. 26, pp. 97-134 (1990)

[5] A. V. Nalitov, L. E. Golub, and E. L. Ivchenko, "Ratchet effects in two-dimensional systems with a lateral periodic potential", Phys. Rev. B 86, 2012.

[6] Sergei V. Koniakhin, "Ratchet effect in graphene with trigonal clusters", Eur. Phys. J. B, 2014, DOI: 10.1140/epjb/e201450434-4.
[7] L.Ermann and D.L.Shepelyansky, "Relativistic graphene ratchet on semidisk Galton board", Physics of Condensed Matter 10/2010; 79(3). DOI: 10.1140/epjb/e2010-10893-1.

[8] A. D. Chepelianskiil, M. V. Entin, L. I. Magarill and D. L. Shepelyansky, "Photogalvanic current in artificial asymmetric nanostructures", Eur. Phys. J. B 56, 323-333, 2007.

[9] J. A. Fürst, J G Pedersen, C Flindt, N A Mortensen, M Brandbyge, T G Pedersen4 and A-P Jauho, "Electronic properties of graphene antidot lattices", New Journal of Physics 11 (2009) 095020 (17pp).

[10] D. Mencarelli, T. Rozzi, L. Pierantoni, "Scattering matrix approach to multichannel transport in many lead graphene nanoribbons", Nanotechnology, Vol. 21, N. 15, Pages: 155701 (10pp), 2010.

[11] D. Medhat, A. Takacs, H. Aubert, J. C. Portal, "Comparative Analysis of Different Techniques for Controlling Ratchet Effect in a Periodic Array of Asymmetric Antidots", Asia Pacific Microwave Conference 2009 (APMC 2009).

[12] D. Mencarelli, L. Pierantoni, T. Rozzi, F. Coccetti, "Nanoscale Simulation of Three-contact Graphene Ballistic Junctions", Nanomater. Nanotechnol. 2014, 4:14. doi: 10.5772/5854.

[13] H.Q. Xu, "Electrical properties of three-terminal ballistic junctions", Appl. Phys. Lett. 78, 2064 (2001).

[14] A. Jacobsen, I. Shorubalko, L. Maag, U. Sennhauser, and K. Ensslin, "Rectification in three-terminal graphene junctions", Appl. Phys. Lett. 97, 032110 (2010); doi: 10.1063/1.3464978.

[15] L. Pierantoni, D. Mencarelli, T. Rozzi, "Boundary Immittance Operators for the Schrödinger-Maxwell Problem of Carrier Dynamics in Nanodevices", IEEE Trans. Microw. Theory Tech., vol. 57, issue 5, pp. 1147-1155, May 2009.

[16] L. Pierantoni, T. Rozzi, "A General Multigrid-Subgridding Formulation for the Transmission Line Matrix Method", IEEE Trans. Microw. Theory Techniques, vol. 55, no. 8, August 2007, pp.1709-1716. 